\title{
Extensional Effects in Viscoelastic Fluid Flow through a Micro-Scale Double Cross-Slot
}

\author{
Mónica S. N. Oliveira ${ }^{a}$, Fernando T. Pinho $^{\mathrm{b}, \mathrm{c}}$ and Manuel A. Alves ${ }^{\mathrm{a}}$ \\ ${ }^{a}$ DEQ, CEFT, Faculdade de Engenharia da Universidade do Porto, R. Dr. Roberto Frias, 4200-465 Porto, Portugal \\ ${ }^{b}$ CEFT, Faculdade de Engenharia da Universidade do Porto, R. Dr. Roberto Frias, 4200-465 Porto, Portugal \\ ${ }^{\complement}$ Universidade do Minho, Largo do Paço, 4704-553 Braga, Portugal
}

\begin{abstract}
The flow of viscoelastic fluids through a microfluidic flow-focusing device was studied numerically. The conceived device is shaped as a double cross-slot with three entrances and three exits, in a symmetric configuration, aiming to achieve a constant extensional rate $(\dot{\varepsilon})$ at the centerline. The two-dimensional numerical simulations using the upper-convected Maxwell (UCM) model allow us to analyze the effect of Deborah number (De) on the flow patterns under creeping flow conditions. As a consequence of the small length scales and high deformation rates associated with the micro-channel flow, strong viscoelastic effects are observed, including purely elastic asymmetries. The double crossslot geometry enables the generation of a region of converging flow, with the added advantage of keeping the flow region of interest away from walls, thus minimizing shear effects. The imposed Hencky strain can be controlled by varying the ratio of the flow rates in the inflowing branches. The shape of the streamlines defining the converging region is strongly influenced by the velocity ratio and by the geometric parameters of the device (e.g. the relative size of the entrance branches). By tuning these parameters, we are able to generate a region of nearly constant $\dot{\varepsilon}$ at the centerline.
\end{abstract}

Keywords: Flow Focusing Device, Cross-Slot, Microfluidics, Extensional Effects, Elastic Instabilities

PACS: 83.50.Jf, 47.15.G, 47.50.Gj, 47.61.-k, 47.11.Df

\section{INTRODUCTION}

The small length scales characteristic of microfluidics enable the generation of flows with high deformation rates while keeping the Reynolds number (Re) small. These unique conditions result in the ability to promote strong viscoelastic effects, which are not concealed by fluid inertia, in fluids that would otherwise exhibit Newtonian-like behavior at the equivalent macroscale. This is particularly important in applications that typically use nonNewtonian fluids such as inkjet printing and lab-on-a-chip devices. There are a number of research studies that take advantage of the small length scales to enhance non-linear rheological effects with different applications in mind [13]. Here, our aim is to develop a device that can be used as an extensional micro-rheometer suitable for measuring extensional properties of dilute polymeric solutions. Towards that goal, we study a double cross-slot device to explore the possibility of attaining a region of constant strain rate. We perform numerical calculations to examine the effect of operating and geometric parameters as well as the effect of De on the flow patterns and velocity field.

\section{NUMERICAL METHOD AND FLOW GEOMETRY}

The double cross-slot device used contains three inlet and three exit channels. The geometry is two-dimensional and symmetric as illustrated in Figure 1, where the main variables are identified. The origin of the coordinate system is set at the center of the entrance cross slot as shown in Figure 1. Side streams are introduced into the central mainstream through two lateral channels of equal dimensions. The width of the lateral channels $\left(D_{2}\right)$ was varied from $0.5 D_{1}$ to $2 D_{1}$, where $D_{1}$ is the width of the central inlet channel. For all configurations tested, the length of the inlet and outlet channels was set to thirty times the central inlet channel width $\left(30 D_{1}\right)$. To account for the effects of geometric parameters, we define the relative width of the entrance branches as $W R=D_{2} / D_{1}$. The average velocity ratio, defined as the ratio of the inlet average velocities in the side streams to the average velocity in the central inlet stream $\left(V R=U_{2} / U_{1}\right)$, was varied between 1 and 200. A flow rate ratio is also used and is defined as $F R=V R W R$.

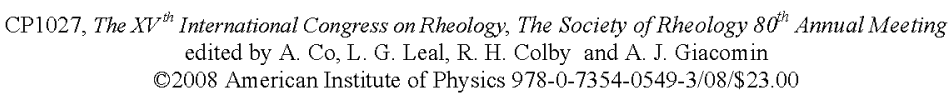


We characterize the degree of elasticity using the Deborah number, $D e \equiv \lambda U_{2} / D_{1}$, where $\lambda$ is the relaxation time of the fluid. For each geometry, De was varied between 0 (corresponding to Newtonian fluid flow) and 0.5 . This was achieved by changing $V R$ and $\lambda$ in the viscoelastic constitutive model. For reasons of rheological simplicity, in this preliminary study we have used the UCM model [4] to describe the viscoelastic fluid, $\boldsymbol{\tau}+\lambda \vec{\tau}=2 \eta \boldsymbol{D}$, where the superscript $\nabla$ denotes the upper-convected derivative, $\tau$ is the extra stress tensor, $\eta$ is the viscosity of the fluid and $D$ is the strain rate tensor.

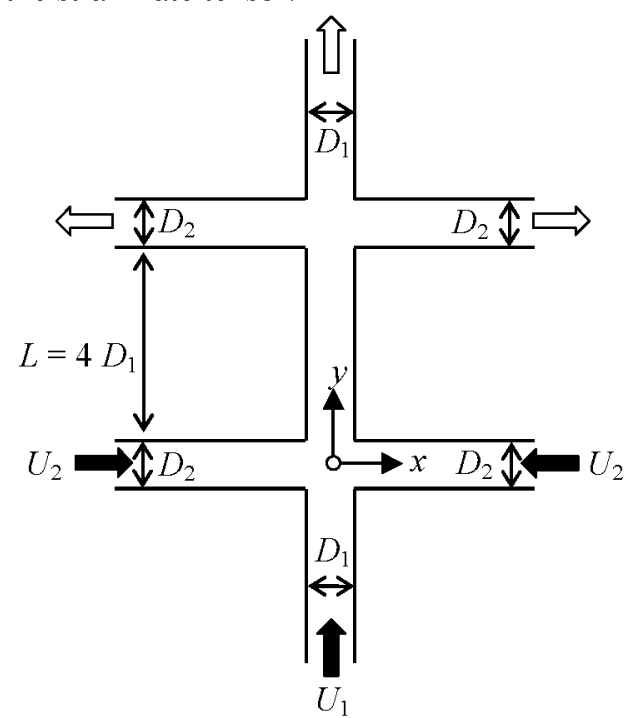

FIGURE 1. Schematics of the double cross-slot geometry.
We consider that the flow is isothermal, incompressible and that it occurs under creeping flow conditions $(R e \rightarrow 0)$. Even though in real flows $R e$ is never zero, it can be considerably small when dealing with microfluidic flows which have characteristic length scales on the order of $10-100 \mu \mathrm{m}$. Thus, by assuming creeping flow in our simulations, we eliminate the effects of inertia and are able to isolate the elastic effects on the flow patterns and the onset of flow asymmetries. In order to model the flow, a fullyimplicit finite volume method is used to solve the appropriate equations of conservation of mass $\nabla \cdot \mathbf{u}=0$ and momentum $-\nabla p+\nabla \cdot \tau=\mathbf{0}$ together with the constitutive equation for $\tau$. A thorough description of the methods used here can be found in [5-6]. We have imposed fully-developed velocity and stress profiles at the inlet boundaries, and Neumann boundary conditions at the outlets. Additionally, the no-slip condition at the walls was imposed.

The spatial domain was mapped using structured and orthogonal meshes that divide the central region of each cross-slot region uniformly into cells of size $\Delta x_{\min }=\Delta y_{\min }=0.02 D_{1}$. The total number of cells (NC) varies according to the specific geometric configuration under study: $N C=27850,40902,66002$ for $W R=0.5,1,2$, respectively.

\section{RESULTS AND DISCUSSION}

Figure 2 illustrates the effect of $V R$ on the flow patterns and the axial velocity profile along the centerline $(x=0)$. As we can see in the axial velocity profiles shown in Figure $2 b$, the fluid accelerates as it approaches the lateral entrances and we can obtain a region of approximately constant strain rate in the converging section, which increases non-linearly with $V R$. One interesting feature of this geometric configuration is that, unlike other geometries [1,7], we can adjust the Hencky strain just by varying $V R$ without changing the size and shape of the device. This is patent in the separation streamlines obtained for different $V R$, shown in Figure 2a. An alternative way to change the Hencky strain is to vary the geometric parameter $W R$. In fact, the controlling parameter is the product of $V R$ and $W R$, i.e. the ratio of flow rates in the inflowing branches, $F R: \varepsilon_{H}=\ln [3(1+2 F R) / 2]$.
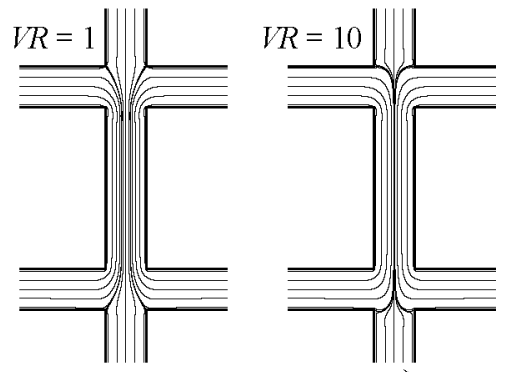

a)

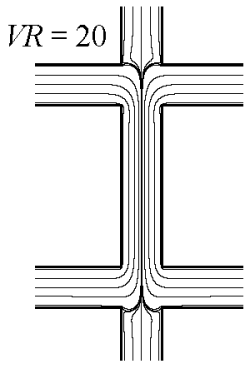

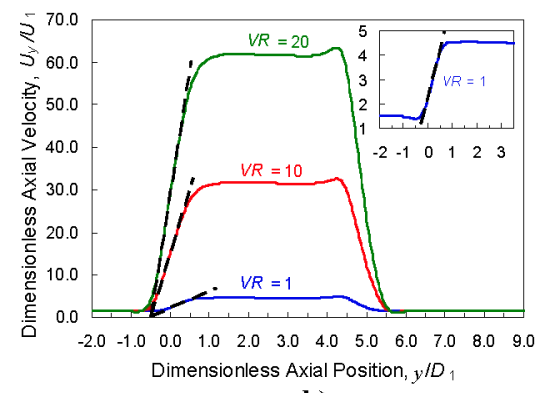

b)

FIGURE 2. Effect of $V R$ on the flow patterns (a) and the axial velocity profiles along the centerline (b) for $W R=1$ and $D e=0.2$.

In Figure 3 we show the effect of De on the axial velocity profiles for different operating and geometric conditions. For high $F R$ (e.g. $F R=20$ ), it is clear that as $D e$ is increased, the flow takes longer to fully develop, and 
there is a reduction of the region where constant $\dot{\varepsilon}$ is observed. Comparing the profiles for $F R=1$, we can see that there is an increase of $\dot{\varepsilon}$ with a decrease of WR. Furthermore, we wish to highlight the undershoot due to diverging streamlines that are present just upstream of the lateral inlet arms and which are enhanced by elasticity, as well as the overshoots at the extremities of the central channel of length $L$. This type of overshoots has been observed in viscoelastic flows through contractions [8] and we see that the overshoot near the inlets is enhanced for lower $W R$ while near the exits is more pronounced for higher $W R$. In both cases, the overshoots are enhanced for higher $D e$.
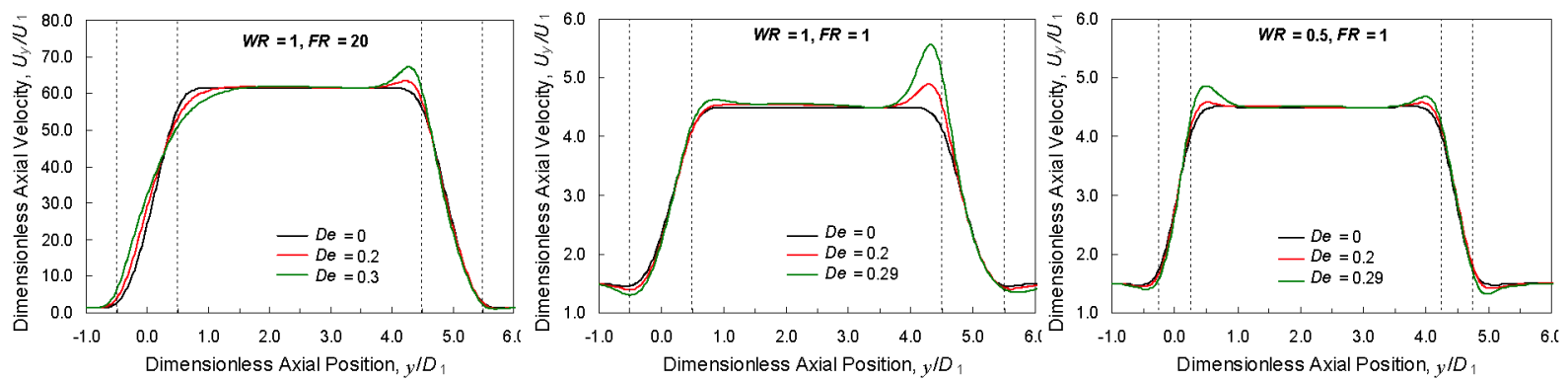

FIGURE 3. Effect of De on the axial velocity profiles along the centerline $x=0$. The dotted lines indicate the lateral channels.

Figure 4 illustrates the effect of $D e$ on the flow patterns, highlighting the development of purely elastic flow asymmetries. For Newtonian and low $D e$ cases $(D e \leq 0.33)$, the flow is steady and symmetric around $x=0$. In this range of $D e$ the curvature of the separation streamlines near the lateral entrances is enhanced as $D e$ is increased. Above a critical $D e, D e \geq 0.34$, the flow remains steady but becomes increasingly asymmetric and bistable, until it eventually becomes time-dependent for $D e \geq 0.5$.

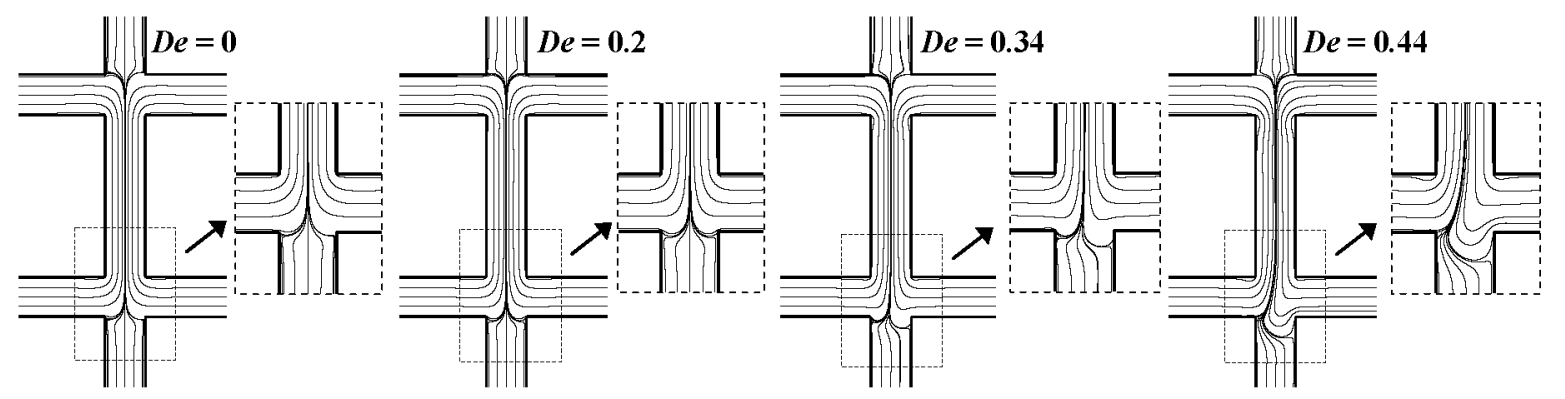

FIGURE 4. The onset of asymmetric flow $(W R=1$ and $V R=20)$.

The transition to asymmetric flow was only observed for sufficiently high $V R, V R \geq 10$. Below this value, the flow evolves from steady and symmetric to unsteady without becoming steady and asymmetric. In the limit of very high $V R$, the critical $D e$ becomes nearly constant, $D e \approx 0.34$. For $W R=0.5$ and 2, qualitatively similar flow patterns are observed but the value of $D e_{c}$ in the limit of high $V R$ is slightly higher $\left(D e_{c} \approx 0.37\right)$ than that obtained for $W R=1$.

\section{ACKNOWLEDGMENTS}

The authors would like to acknowledge FCT and FEDER for funding through projects PPCDT/EME/59338/2004 and PTDC/EQU-FTT/7 1800/2006.

\section{REFERENCES}

1. L. E. Rodd, T. P. Scott, D. V. Boger, J. J. Cooper White and G. H. McKinley, J. Non-Newt. Fluid Mech. 129, 1-22 (2005).

2. J. S. Lee, R. Dylla-Spears, N. P. Teclemariam, and S. J. Muller, Appl. Phys. Lett. 90, 074103 (2007).

3. T. M. Squires and S. R. Quake, Rev. Mod. Phys. 77, 977-1026 (2005).

4. J. G. Oldroyd, Proc. R. Soc. A 200, 523-541 (1950).

5. P. J. Oliveira, F. T. Pinho and G. A. Pinto, J. Non-Newt. Fluid Mech. 79, 1-43 (1998).

6. M. A. Alves, P. J. Oliveira and F. T. Pinho, J. Non-Newt. Fluid Mech. 41, 47-75 (2003).

7. M. S. N. Oliveira, M. A. Alves, F. T. Pinho and G. H. McKinley, Exp. in Fluids 43, 437-451 (2007).

8. M. A. Alves and R. J. Poole, J. Non-Newt. Fluid Mech. 144, 140-148 (2007). 